\title{
Thermoplasmonic ssDNA dynamic release from gold nano- particles examined with advanced fluorescence microscopy
}

\author{
Sabrina Simoncelli, ${ }^{1,2 *}$ Hasitha de Alwis Weerasekera, ${ }^{1 *}$ Chiara Fasciani, ${ }^{1}$ Christopher Boddy, ${ }^{1}$ Pedro F. Ara- \\ mendia, ${ }^{2}$ Emilio I. Alarcon ${ }^{1 *+}$ and Juan C. Scaiano ${ }^{1 *}$ \\ ${ }^{1}$ Department of Chemistry and Centre for Catalysis Research and Innovation, University of Ottawa, Ottawa, Ontario, K1N 6N5. \\ ${ }^{2}$ INQUIMAE and Departamento de Química Inorgánica, Analítica y Química Física, Facultad de Ciencias Exactas y \\ Naturales, Universidad de Buenos Aires, Pabellón 2, Ciudad Universitaria, 1428 Buenos Aires, Argentina.
}

Supporting Information Placeholder

\begin{abstract}
Plasmon excitation of spherical gold nanoparticles carrying a fluorescent labeled $30 \mathrm{bp}$ dsDNA cargo, with one chain covalently attached through two $\mathrm{S}$-Au bonds to the surface, results in release of the complementary strand as ssDNA that can be examined in situ using high-resolution fluorescence microscopy. The release is dependent on the total energy delivered, but not the rate of delivery, an important property for plasmonic applications in medicine, sensors and plasmon induced PCR.
\end{abstract}

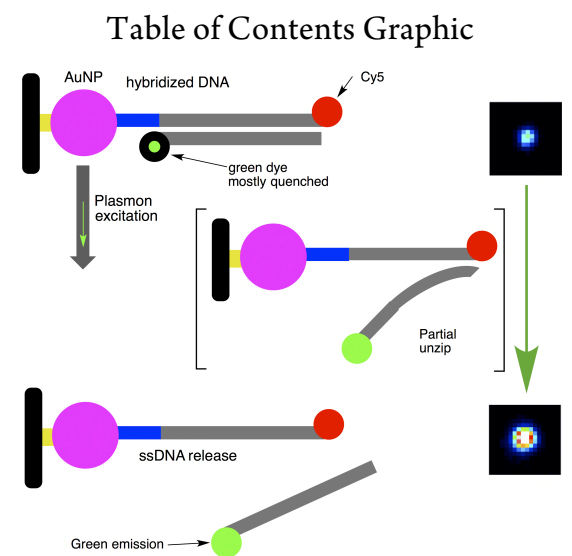

Keywords: Plasmonics, DNA, nanotechnology, gold nanoparticles

Plasmon mediated heating involves localized ultrafast energy-to-heat conversion upon light interaction with electrons of a given metal nanoparticle. ${ }^{1}$ In the case of gold nanoparticles (AuNP) this heat can increase the temperature hundreds of degrees near the surface of the nanoparticle, for submicrosecond times following laser excitation. ${ }^{2}$ While thermoplasmonic effects in long time scales are determined by the principles of thermodynamics and the dynamics of heat dissipation ${ }^{1}$ and have been recently utilized for compact autoclaves, ${ }^{3}$ or in photothermal imaging for single particle detection ${ }^{4}$ at very short time scales molecules can experience high-temperature behavior that reflects their location and interaction with the plasmonic field.

Realistic technological applications of plasmon heating in devices based on nanostructures will require enabling chemical platforms to support the efficient energy delivery without affecting the surface of the nanoparticle. Here, we present a new platform; an on-off fluorescence switch is used to report single strand DNA (ssDNA) release from AuNP, where only one of the chains of double strand DNA (dsDNA) is covalently attached to the nanoparticle. Meanwhile the second chain, attached only through DNA hybridization, is released upon plasmonic excitation. Beyond sensors ${ }^{5}$ and therapeutic applications ${ }^{6,7}$ we foresee a potential for polymerase chain reaction (PCR) applications with a dramatic reduction of bulk heating requirements. Since thermoplasmonic strategies can deliver energy with pinpoint precision while the bulk solution remains at near ambient temperature, this will allow the developments of novel applications such as remote controlled in-vitro PCR. In the case of potential photoplasmonicPCR applications, a strategy that allows reversible binding to the AuNP surface would have to be developed; at the same time, the complexity of a design around available fluorescent dyes and laser wavelengths would not be required.

Branda et al..$^{8-10}$ and Halas et al., ${ }^{11-13}$ have pioneered the field of plasmon-mediated release. Reports include the release of ssDNA with a complementary strand bound to a gold nanoparticle in solution. ${ }^{10,11,14}$ In Branda's approach they linked their complementary $15 \mathrm{bp}$ strand through a $5^{\prime}$ terminal $-\mathrm{SH}$ anchor $^{15}$ to the nanoparticle surface. A threshold of $\approx 0.5$ $\mathrm{W} / \mathrm{cm}^{2}$ was found as the limit for breaking the S-Au bond. Plasmon induced release was also observed by Halas et al. ${ }^{11}$,

${ }^{13}$ One example has also been reported where local plasmonic heating hinders hydridization. ${ }^{16}$ In the present communication, we examine the dynamics of ssDNA plasmon release by using an anchoring system composed of lipoic acid (LA), a molecule that has been employed as oligo-linker for gold nanoparticles, ${ }^{17,18}$ and a five-carbon spacer to a $30 \mathrm{bp}$ duplex, see Scheme 1. We note that in this case ssDNA release does not involve covalent bond cleavage; further, the use of LA 
provides two S-Au links minimizing the risk of cleavage at the AuNP surface. Single strand release from the nanoparticle surface was in-situ monitored for the first time using highresolution microscopy resolving emissive events and with spatial resolution at subcellular dimensions.

Surface bonded sequence (via lipoic acid) 5'- /5AmMC6/ AACACAAACACACTTAAACACAAACACACC /3Cy5N/-3'

Free sequence 5'- GGTGTGTTTGTGTTTAAGTGTGTTTGTGTT /3ATTO550N/ -3'

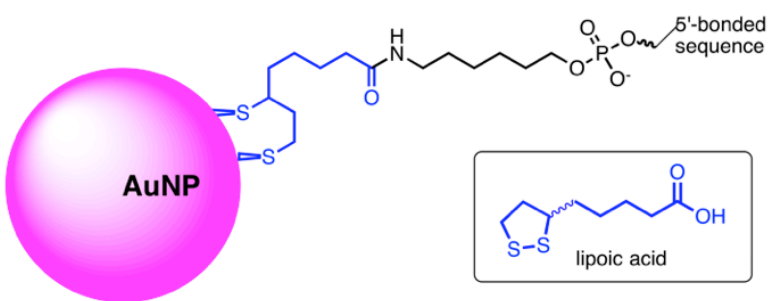

Scheme 1: Details of the sequences employed and nature of the lipoic binding to AuNP.

Scheme 2 shows a representation of the experimental design. 3-Aminopropyltriethoxisilane (APTES) was used to attach the spherical $22 \pm 3 \mathrm{~nm}$ AuNP onto the glass surface (see section S2, Fig. S1 and S2). LA anchors through its two-thiol functionalities to the gold surface, Scheme 1; the "two-feet" surface binding prevents the release of dsDNA (with the Cy5 dye as a control, see Fig. 1). Further, LA also serves as a spacer and anchor through its carboxylic moiety to the 5'amine strand in the 30 bp dsDNA shown in Scheme 1. The dsDNA was chemically attached to the gold surface, ranging between 200-500 dsDNA per nanoparticle (see SI), where the uncertainty reflects the allowance for the spacer that effectively provides some relief for DNA crowding on the surface. These characteristics are critical for our real-time fluorescence detection. ATTO550, which is located at $\sim 2.0 \mathrm{~nm}$ from the AuNP surface in the bonded oligo-strand would be largely quenched and retain only $5.0-20 \%$ of its original brightness. Meanwhile, for Cy5, which is located $\sim 8.0 \mathrm{~nm}$ from the metal surface would show $70-90 \%$ of its original brightness (see section S3, Fig. S3 and S4). Our strategy uses Cy5 as a reporter to quantify loss of DNA binding due to potential, but highly unlikely, cleavage of the LA-AuNP linkage, see Scheme 2 .

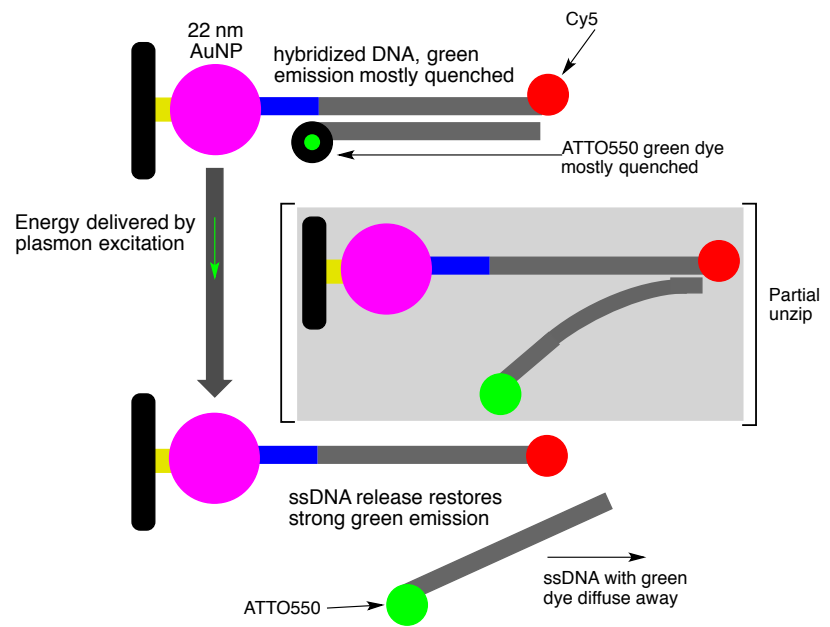

Scheme 2: Representation of dsDNA-AuNP conjugate system. dsDNA is attached through an amide bond between the bonded strand, $\mathrm{Cy} 5$ labeled, and the lipoic acid (in blue) functionalized AuNP. On the left, the black bar symbolizes the glass surface and APTES attachment (yellow). Upon plasmon excitation release of the ssDNA label with ATTO550 takes place with a concomitant increase in the fluorescence intensity (see section $\mathrm{S3}$ ). Covalent bond cleavage is not involved in the release process. The square brackets show a partially unzipped system, a necessary step in the release dynamics.

Total internal reflection fluorescence (TIRF) images, obtained with 543 or $633 \mathrm{~nm} \mathrm{CW}$ laser excitation, for the samples containing the dsDNA attached to the AuNP were acquired. Image sequences taken up to $250 \mathrm{~s}$ using $543 \mathrm{~nm}$ excitation showed regions with intense emissions, whose intensities and size change as a function of the exposure time and energy (see Fig. 2A for intensity changes for representative bright spots over the AuNP functionalized slide). Further, emission spectra and lifetimes for the fluorescent spots matched those of ATTO550 in solution as seen in Fig. 2B. Experiments were performed at $633 \mathrm{~nm}$ to test $\mathrm{Cy} 5$ photostability, only produced bleaching of the AuNP-bound ssDNA Cy 5 emission to a similar extent to the observed for the free construct in solution (see Fig. S5 and S6).

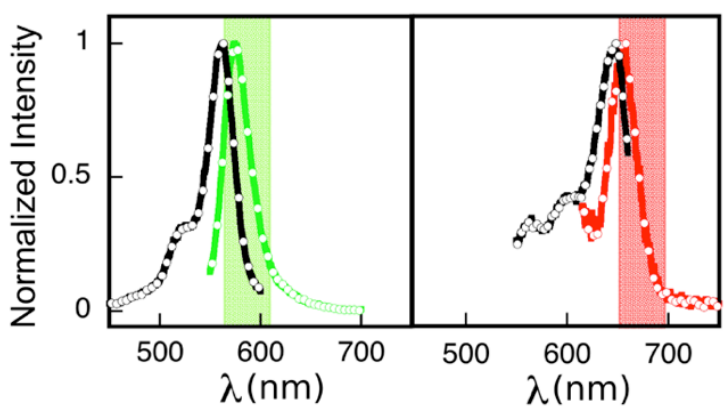

Figure 1: Excitation (black line) and emission (colored lines) spectra of the dsDNA labeled with ATTO550 (green, left pan- 
el) and Cy5 (red, right panel). The color-shaded areas represent the bandpass filters for the fluorescence emission.

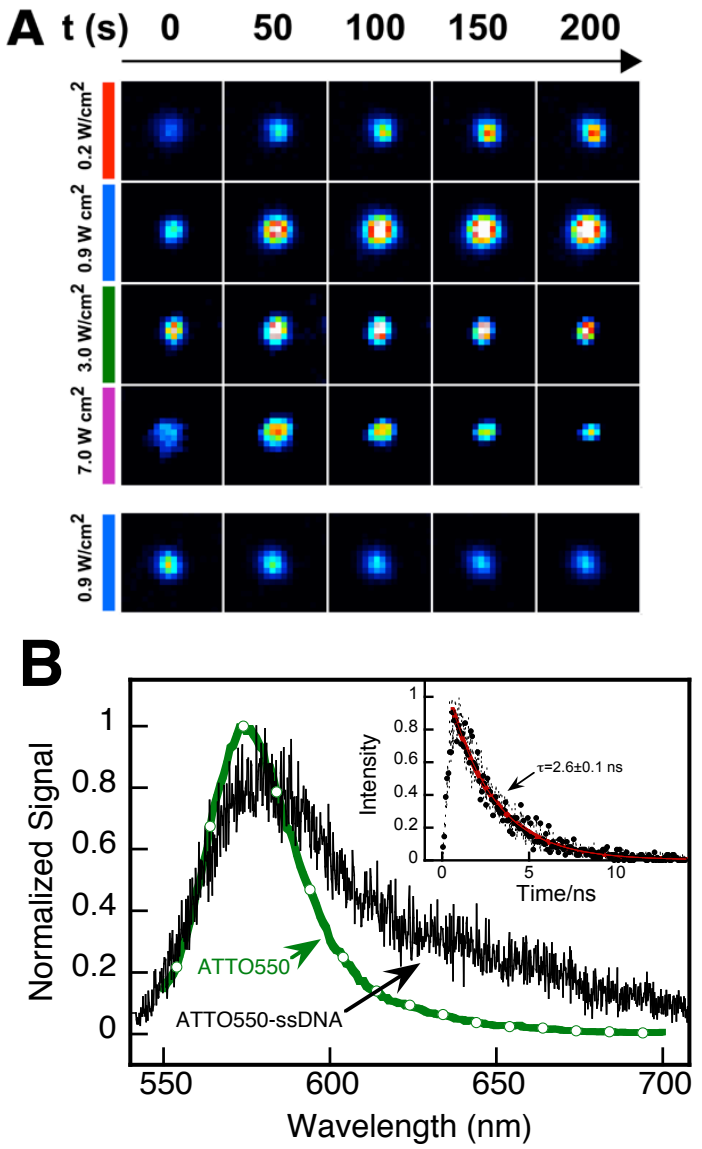

Figure 2. Real-time thermoplasmonic release of ssDNA from AuNP monitored in-situ by TIRF microscopy using a $543 \mathrm{~nm}$ CW laser. (A) Representative fluorescence intensity images of ssDNA-ATTO550 release as a function of the irradiation time and the irradiance. The area of each image is $9 \mu \mathrm{m}^{2}$ (pixel size $156 \mathrm{~nm}$ ). The image uses a LUT false color scheme to represent the intensity of the fluorescence signal. The bottom sequence in panel A corresponds to a control using ATTO550@AuNP. (B) Emission spectra for ATTO550 free (green) and released from the dsDNA-AuNP conjugates (black) upon plasmon excitation. Inset: ATTO550 fluorescence decay under the same experimental conditions $\left(\tau_{\mathrm{f}} \sim 2.6 \mathrm{~ns}\right.$, data fit is shown in red).

The increase in signal intensity of ATTO550 can be interpreted as the specific release of the ATTO-labeled (i.e., the non-covalently bonded) strand from the quenching zone of the AuNP as depicted in Scheme 2. Physical desorption from the gold surface can be ruled out as an important factor responsible for the ATTO550 emission changes, since experiments with non-complementary ATTO550 labeled oligonucleotides or free ATTO550 dye showed no emission changes (data not shown). To further verify that the release of non-covalently bonded ssDNA takes place and not of the bonded dsDNA, control experiments were carried out. First, a $530 \mathrm{~nm}$ LED as an illumination source $\left(0.32 \mathrm{~W} / \mathrm{cm}^{2}\right)$ was used over the dsDNA@AuNP slides revealing only the presence of ATTO550 fluorescence with non-detectable $\mathrm{Cy} 5$ release after $10 \mathrm{~min}$ irradiation, see section S4 SI. Second, LA-AuNP slides were functionalized with an aminoATTO550 dye such that the dye was covalently attached to the AuNP (ATTO550@AuNP) (Fig. 2A, 3A and S7). Imaging of this sample in the TIRF system, showed only photobleaching of the ATTO550 dye, presumably by interaction with the excited AuNP; in this case the proximity is such that electron transfer (i.e., exchange) processes may contribute to degradation. Thus, the increments in emission are fully consistent with a release mechanism due to specifically plasmoninduced melting of the non-covalent dsDNA interaction.

It is interesting to compare the intensity of the fluorescence spots in Figure 2A with the intensity curves in Figure 3A, bearing in mind that Figure 2A shows only representative spots, rather than average or ensemble data. This is particularly relevant for the series at 3.0 and $7.0 \mathrm{~W} / \mathrm{cm}^{2}$. In both cases the second image (at $50 \mathrm{~s}$ ) is the more intense one, but at $7.0 \mathrm{~W} / \mathrm{cm}^{2}$ the loss of intensity is faster. The second image in Figure 2A corresponds to 150 and $350 \mathrm{~J} / \mathrm{cm}^{2}$ for, 3.0 and $7.0 \mathrm{~W} / \mathrm{cm}^{2}$ respectively. The corresponding curves in Figure $3 \mathrm{~A}$ show maxima in the $200-250 \mathrm{~J} / \mathrm{cm}^{2}$ region in agreement with the images in Figure 2A, where the last image for 3.0 and $7.0 \mathrm{~W} / \mathrm{cm}^{2}$ corresponds to 600 and $1400 \mathrm{~J} / \mathrm{cm}^{2}$, respectively; thus, it is not surprising that the latter shows faster bleaching or degradation.
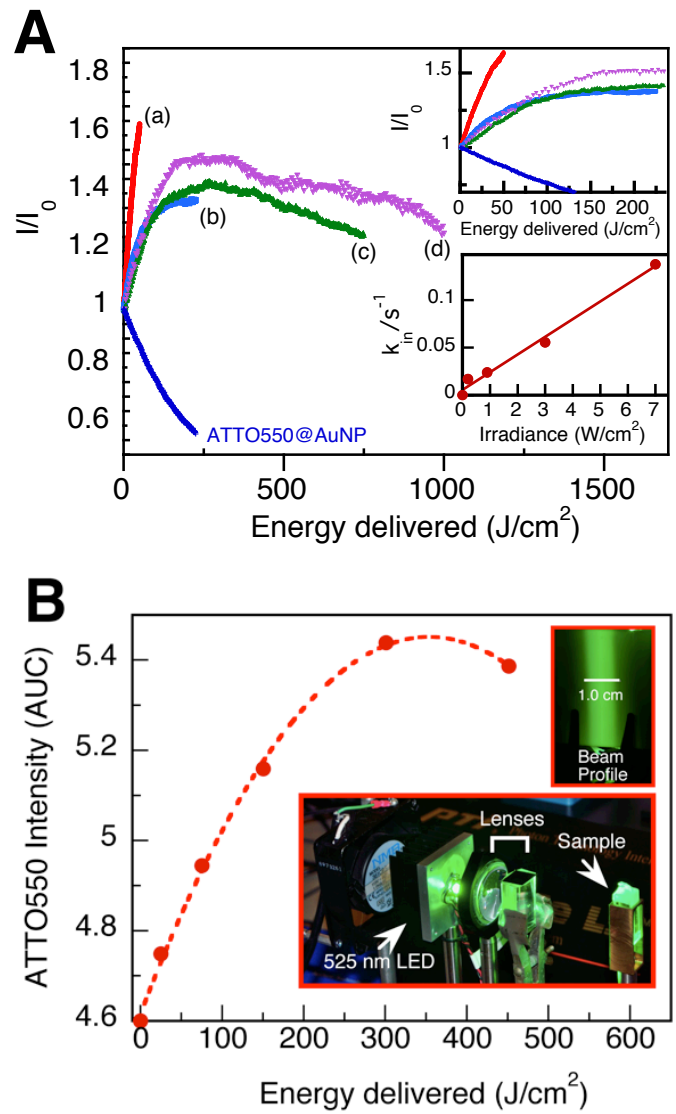
Figure 3. (A) Fluorescence intensity enhancement for ssDNAATTO550 release events as a function of total energy delivered. Average between 50-90 intensity vs. time curves measured over single bright spots (see Fig. 2) are plotted for each of the irradiances (a) red 0.2, (b) blue 0.9, (c) green 3.0, and (d) purple 7.0 $\mathrm{W} / \mathrm{cm}^{2}$. ATTO550@AuNP conjugates were also tested as a control experiment $\left(0.9 \mathrm{~W} / \mathrm{cm}^{2}\right.$, showing only emission decrease). Top inset: Expansion of the low energy region for Figure 3A. Bottom inset: Initial slope of the fluorescence intensity vs. time for the experiments of Figure $3 \mathrm{~A}$ as function of the irradiance (curves shown in Fig. S8). (B) ATTO550-ssDNA emission measured upon LED exposure of dsDNA@AuNP 1x1 cm quartz cuvettes (see section S4, SI). Top inset: shows the beam distribution of employed in our experimental setup (see bottom inset in this Figure).

In-situ thermoplasmonic dsDNA melting dynamics were explored analyzing 50-90 bright spots from three independent experiments, ${ }^{19}$ measured at different $543 \mathrm{~nm}$ CW laser irradiances, ranging from 0.2 to $7.0 \mathrm{~W} / \mathrm{cm}^{2}$. Fig. $\mathrm{S} 8$ shows that ssDNA release becomes faster as the laser irradiance increases, while Figure 3A shows dependence with the total dose delivered. However, the top inset of Fig. 3A shows that for irradiances at/or above $0.9 \mathrm{~W} / \mathrm{cm}^{2}$ uniquely that only the total energy matters and not the form or rate of delivery. Only at very low irradiances (curve (a) in Fig. 3A) is there a deviation from this behavior, as seen in the bottom inset of Fig. 3A, with very low intensities being slightly more effective. This suggests a minor abundance $(<2 \%)$ of an easily released population that is activated at low energies, ${ }^{20}$ but is too small to affect the overall observations at higher irradiances, where specific release due to cleavage of the noncovalent dsDNA interaction dominates. Further, $525 \mathrm{~nm}$ LED $\left(22 \mathrm{~mW} / \mathrm{cm}^{2}\right)$ in-situ release experiments were carried out in $1 \times 1 \mathrm{~cm}$ fluorescence cuvettes whose walls where modified with AuNP and the dsDNA, see section S4, SI. These experiments showed similar behavior in terms of ATTO550 fluorescence intensity $v s$. delivered power and those obtained at the single particle level, Fig. 3B, with non-detectable Cy5 emission.

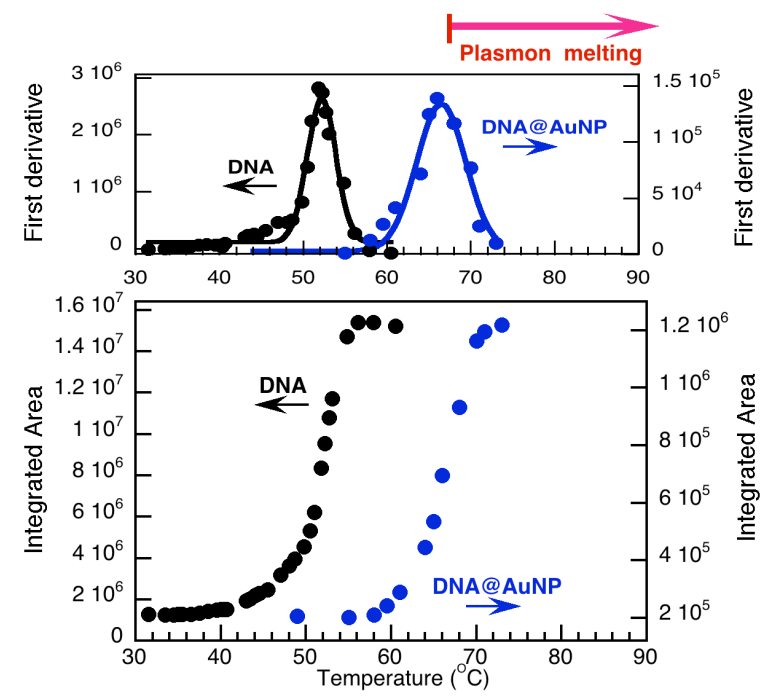

Figure 4. Melting curves for dsDNA in solution and when bound to AuNP using the strategy of Scheme 2. The top panel shows first derivative plots. The data correspond to the fluorescence increase as the temperature is raised. Note that the intensity values are smaller for DNA@AuNP (right), a measure of the low amount of DNA available at the surface and the difficulties of this measurement.

The melting temperature $\left(\mathrm{T}_{\mathrm{m}}\right)$ of the dsDNA used in this study was $52 \pm 2^{\circ} \mathrm{C}$ at $10 \mathrm{mM} \mathrm{NaCl}$; interestingly, this value increases to $66 \pm 1^{\circ} \mathrm{C}$ when the same dsDNA is bound to the AuNP surface, which suggests an increment in the DNA packing onto the nanoparticle surface. Such of an increment in $T_{m}$ is consistent with earlier studies ${ }^{13}$ (Fig. 4). Clearly, upon plasmonic excitation, the dsDNA bound to the AuNP shows effects similar to those observed at bulk temperatures that exceed $66^{\circ} \mathrm{C}$. The fact that the slopes of release curves at irradiances $>0.9 \mathrm{~W} / \mathrm{cm}^{2}$ are essentially identical, which suggests that the release mechanism does not change, and is fully compatible with the ssDNA release discussed above. Thus, each photon arrival to a particle (approximately every $100 \mathrm{~ns}$ at $\sim 1 \mathrm{~W} / \mathrm{cm}^{2}$ ) has a constant probability of causing ssDNA release before the energy dissipates. Baffou and Quidant $^{1,21}$ estimated that a $20 \mathrm{~nm}$ AuNP in water will experience a steady state $5^{\circ} \mathrm{C}$ increase in temperature when exposed to $1 \mathrm{~mW} / \mu^{2}$ at $530 \mathrm{~nm}$. Our irradiances are much lower than this, and the steady state temperature change should be well below $1^{\circ} \mathrm{C}$, thus insufficient to cause dsDNA dissociation. Thus, ssDNA release is not caused by bulk heating of the solution, but rather it is a highly efficient surface local event. ${ }^{22}$ It is possible that release events are favored at inter-particle locations (hot plasmonic spots) as it occurs in the case of plasmon-induced polymerization. ${ }^{23,24}$

Further measurements at increasing salt concentrations did not show any statistical significance even at $1.2 \mathrm{M} \mathrm{NaCl}$ salt concentration. ${ }^{25,}{ }^{26}$ It seems likely that for successful release events the temperature sensed by DNA is such that moder- 
ate changes in melting temperature do not change the release efficiency, instead it seems that the packing of the dsDNA molecules onto the gold surface directs the melting dynamics of the oligo-nucleotide.

In summary, we report the in-situ dynamics of thermoplasmonic ssDNA release from AuNP surface using fluorescence microscopy. Our findings point to the suitability of LA as a superior anchor able to support plasmon heating for ssDNA release. ${ }^{10}$ Our data indicate that the thermoplasmonic rate of ssDNA release is tunable by varying the excitation irradiance, and that -within the range examined-release is determined by the total energy delivered, and not by the rate of delivery; quite significantly, this implies that while laser sources are convenient tools for our research, practical applications of this technology could be performed with simpler (and less expensive and safer) light sources, such as LEDs. Further, linearity with light source $\mathrm{CW}$ power is an asset for potential applications in sensors, therapeutics, or potential room temperature miniaturized PCR devices. It is exciting to think about temporal and spatial control of PCR as these are common characteristics for photochemical processes. Would having the materials attached to a nanoparticle enable cellspecific PCR? If the nanoparticle provides protection and a method for crossing cell membranes strategies could be developed to release photochemically siRNA in biological systems; a recent report suggests that this is indeed possible. ${ }^{12}$ The demonstration of photo-controlled thermoplasmonic melting of DNA suggests that those possibilities are within reach.

Supporting Information: Experimental details, characterization of AuNP on slides, DNA melting details and effect of power on acquired images. This material is available free of charge via the Internet at http://pubs.acs.org."

\section{AUTHOR INFORMATION}

Inquiries to: scaiano@photo.chem.uottawa.ca

\section{Author Contributions}

$\$$ These authors contributed equally. ${ }^{\dagger}$ Current address: University of Ottawa Heart Institute, Bio-nanomaterials Chemistry and Engineering laboratory (BnCE).

\section{ACKNOWLEDGMENT}

JCS and CB thank NSERC for support of this research. SS thanks CONICET for a PhD Scholarship and UBA for support. A DFAIT (Canada) fellowship from ELAP (Emerging Leaders in the Americas Program) supported SS's Canada visit.

\section{REFERENCES}

(1) Baffou, G.; Quidant, R., Thermo-plasmonics: using metallic nanostructures as nano-sources of heat. Laser \& Photonics Rev. 2013, 7, (2), 171-187.

(2) Fasciani, C.; Bueno Alejo, C. J.; Grenier, M.; Netto-Ferreira, J. C.; Scaiano, J. C., High-Temperature Organic Reactions at Room Temperature Using Plasmon Excitation: Decomposition of Dicumyl Peroxide. Org. Lett. 2011, 13, (2), 204-207.
(3) Neumann, O.; Feronti, C.; Neumann, A. D.; Dong, A.; Schell, K.; Lu, B.; Kim, E.; Quinn, M.; Thompson, S.; Grady, N.; Nordlander, P.; Oden, M.; Halas, N. J., Compact solar autoclave based on steam generation using broadband light-harvesting nanoparticles. PNAS 2013, 110, (29), $11677-11681$

(4) Chang, W.-S.; Ha, J. W.; Slaughter, L. S.; Link, S., Plasmonic nanorod absorbers as orientation sensors. PNAS 2010, 107, (7), 2781-2786.

(5) Coll, C.; Bernardos, A.; Martínez-Máñez, R.; Sancenón, F., Gated Silica Mesoporous Supports for Controlled Release and Signaling Applications. Acc. Chem. Res. 2013, 46, (2), 339-349.

(6) Huang, X.; El-Sayed, I. H.; Qian, W.; El-Sayed, M. A., Cancer Cell Imaging and Photothermal Therapy in the Near-Infrared Region by Using Gold Nanorods. J. Am. Chem. Soc. 2006, 128, (6), 2115-2120.

(7) Huang, X. H.; Jain, P. K.; El-Sayed, I. H.; El-Sayed, M. A., Determination of the minimum temperature required for selective photothermal destruction of cancer cells with the use of immunotargeted gold nanoparticles. Photochem. Photobiol. 2006, 82, (2), 412-417.

(8) Bakhtiari, A. B. S.; Hsiao, D.; Jin, G.; Gates, B. D.; Branda, N. R., An Efficient Method Based on the Photothermal Effect for the Release of Molecules from Metal Nanoparticle Surfaces. Angew. Chem. Int. Ed. 2009, 48, (23), 4166-4169.

(9) Yan, B.; Boyer, J.-C.; Habault, D.; Branda, N. R.; Zhao, Y., Near Infrared Light Triggered Release of Biomacromolecules from Hydrogels Loaded with Upconversion Nanoparticles. J. Am. Chem. Soc. 2012, 134, (40), 16558-16561.

(10) Poon, L.; Zandberg, W.; Hsiao, D.; Erno, Z.; Sen, D.; Gates, B.; Branda, N., Photothermal release of single-stranded DNA from the surface of gold nanoparticles through controlled denaturating and Au-S bond breaking. ACS nano 2010, 4, (11), 6395-6403.

(11) Barhoumi, A.; Huschka, R.; Bardhan, R.; Knight, M. W.; Halas, N. J., Light-induced release of DNA from plasmon-resonant nanoparticles: Towards light-controlled gene therapy. Chem. Phys. Lett. 2009, 482, (46), 171-179.

(12) Huschka, R.; Barhoumi, A.; Liu, Q.; Roth, J. A.; Ji, L.; Halas, N. J., Gene Silencing by Gold Nanoshell-Mediated Delivery and LaserTriggered Release of Antisense Oligonucleotide and siRNA. ACS Nano 2012, 6, (9), 7681-7691.

(13) Huschka, R.; Zuloaga, J.; Knight, M. W.; Brown, L. V.; Nordlander, P.; Halas, N. J., Light-Induced Release of DNA from Gold Nanoparticles: Nanoshells and Nanorods. J. Am. Chem. Soc. 2011, 133, (31), 12247-12255

(14) Reismann, M.; Bretschneider, J. C.; Plessen, G. v.; Simon, U., Reversible Photothermal Melting of DNA in DNA-Gold-Nanoparticle Networks. Small 2008, 4, (5), 607-610.

(15) The following sequences are reported by Branda et al: 5'TTTCATAGTTGACCTCT3' (Sense); 5'AGAGGTCTACTATG3' (Antisense). The correct antisense sequence (that was actually used) is: 5'AGAGGTCAACTATG3'. We thank Professor Branda for confirming the use of this sequence.

(16) Osinkina, L.; Carretero-Palacios, S.; Stehr, J.; Lutich, A. A.; Jäckel, F.; Feldmann, J., Tuning DNA Binding Kinetics in an Optical Trap by Plasmonic Nanoparticle Heating. Nano Lett. 2013, 13, (7), 3140-3144.

(17) Barrett, L.; Dougan, J. A.; Faulds, K.; Graham, D., Stable dyelabelled oligonucleotide-nanoparticle conjugates for nucleic acid detection. Nanoscale 2011, 3, (8), 3221-3227.

(18) Guerrini, L.; Barrett, L.; Dougan, J. A.; Faulds, K.; Graham, D., Improving the understanding of oligonucleotide-nanoparticle conjugates using DNA-binding fluorophores. Nanoscale 2013, 5, (10), 4166-4170.

(19) We prepared three separate samples of dsDNA@AuNP conjugates and measured each of them in different sections using four different laser irradiances. We identified 20 to 30 bright spots in the video, leading to 60 to 90 spots (such as those in Figure 2A) and we calculated the intensity vs. time for each spot, averaging the results, leading to the Data in Figure 2C. 
(20) We speculate that the $2 \%$ of relatively easy release may be due to a slight stoichiometric mismatch, i.e. a slight excess of the ATTO labeled chain. This chain could not hybridize due to the slight deficit of the complementary chain. It could still have some affinity for the gold surface, but be readily released upon thermoplasmonic heat-ing well below the melting temperature of the assembly of Scheme 2 .

(21) Baffou, G.; Quidant, R.; GarcÃıa de Abajo, F. J., Nanoscale Control of Optical Heating in Complex Plasmonic Systems. ACS Nano 2010, 4, (2), 709-716.

(22) We have considered a step type gradient model, which involves the homogeneous heating of a $10 \mathrm{~nm}$ layer around the AuNP that has reached a thermal stationary state for the heat release.

(23) Stamplecoskie, K. G.; Pacioni, N. L.; Larson, D.; Scaiano, J. C., Plasmon-Mediated Photopolymerization Maps Plasmon Fields for Silver Nanoparticles. J. Am. Chem. Soc. 2011, 133, (24), 9160-9163.
(24) Scaiano, J. C.; Stamplecoskie, K., Can Surface Plasmon Fields Provide a New Way to Photosensitize Organic Photoreactions? From Designer Nanoparticles to Custom Applications. The Journal of Physical Chemistry Letters 2013, 4, (7), 1177-1187.

(25) Owczarzy, R.; Moreira, B. G.; You, Y.; Behlke, M. A.; Walder, J. A., Predicting Stability of DNA Duplexes in Solutions Containing Magnesium and Monovalent Cations. Biochem. 2008, 47, (19), 53365353.

(26) Owczarzy, R.; You, Y.; Moreira, B. G.; Manthey, J. A.; Huang, L.; Behlke, M. A.; Walder, J. A., Effects of Sodium Ions on DNA Duplex Oligomers: Improved Predictions of Melting Temperatures. Biochemistry 2004, 43, (12), 3537-3554. 